
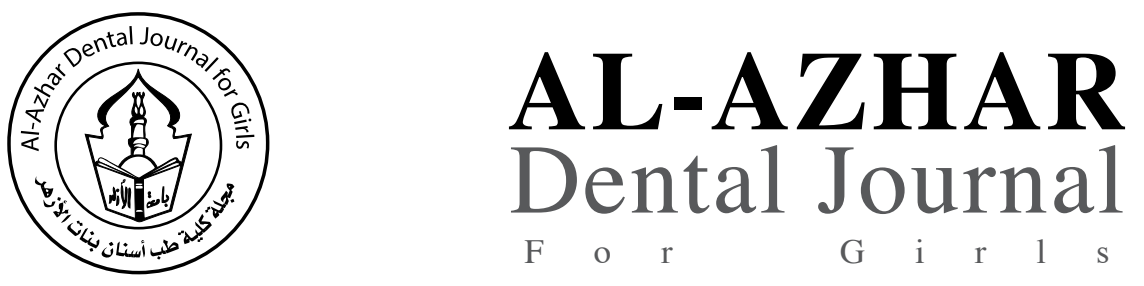

The Official Publication of The Faculty of Dental Medicine For Girls,

Al-Azhar University Cairo, Egypt.

ADJ-for Grils, Vol. 3, No. 3, July (2016) — PP. 183:191

\title{
Effect of Hydroxyapatite and Sodium Hexameta Phosphate Application on Demineralized Enamel and Dentine
}

\author{
Sara O. Zarem ${ }^{(1)}$, Maha A. Niazy ${ }^{(2)}$ and Nevein A. Gad ${ }^{(3)}$
}

Codex : 04/1607

dentaljournal.forgirls@yahoo.com

\section{KEYWORDS}

Hydroxyapatite,

sodium hexametaphosphate,

Energy Despersive

$X-\operatorname{ray}(E D X A)$,

scanning electron microscope

(SEM).

\begin{abstract}
The aim of the present study was to evaluate the remineralizing effect of (hydroxyapatite nano particles,sodium hexametaphosphate, hydroxyapatite nanoparticles and sodium hexametaphosphate in combination) with and without use of artificial saliva on enamel and dentin. Forty freshly extracted human premolars were used in this study. They should be free from caires, macroscopic cracks, abrasion or extensive staining. The teeth were kept in normal saline until use. Each tooth was horizontally cut to separate the crown from the root by using a low - speed double faced diamond disc. Each crown were then sectioned longitudinally in to two parts (buccal and lingual) then put it in normal saline to avoid dehydration.The samples were divided into (2) main groups of (15) samples, where group one consist of (15) teeth (2 halves) not immersed in artificial saliva, while group two included the samples which immersed in artificial saliva. According to the substrate tissue they devided into enamel group (B1) and dentine group (B2). Each group was then subdivided into four subgroups of 5 samples each, according to the treatment solution, Subgroup (A1): treated with hydroxyapatite nanoparticle, Subgroup (A2): treated with sodium hexametaphosphate and Subgroup (A3): treated with combination of two materials (HA + SHMP).Sample were subjected to the following testing procedure: 1) The qualitative micro-morphological changes that occurred in enamel surface and in the dentin after etching and after the application of the remineralizing solutions.2)The quantitative analyzed using Energy dispersive X-ray spectrometric Analysis (EDXA) to determine the calcium and phosphorous mineral content and the change in the levels of these elements percentage due to demineralization. As well, the fluoride content was deduced in respect to these subgroups treated with HA solutions.The results was found that $(\mathbf{H A}+\mathbf{S H M P + A S})$ group in enamel recorded the highest total mean mineral contents, followed by $(\mathbf{H A}+\mathbf{A S})$ group in enamel, then group (HA+SHMP) in dentine, while the mean for control (HA) in enamel group recorded the lowest total mean mineral contents. The difference between groups was statistically
\end{abstract}

1. B.D.S, Faculty of Oral and Dental Medicine, Tripoly University(2008). Professor and Head of Operative Dentistry Department. Faculty of Dental Medicine for Girls, Al-Azhar University.

2. Professor and Head of Operative Dentistry Department. Faculty of Dental Medicine for Girls, Al-Azhar University.

3. Lecturer of Operative Dentistry Department. Faculty of Dental Medicine for Girls, Al-Azhar University. 
in -significant. Also in SEM micrographs showed that enamel and dentin surface which treated with different solutions, the amount of deposits differed depending of type of solution used. It was concluded that Hydroxyapatite nanoparticle has a better effect on demineralized enamel when used with artificial saliva while the Control hydroxyapatite solution recorded the lowest mineral precipitation and remineralization formed on surface of demineralized enamel dentin substrate and Sodium hexametaphosphate has a significant role in mineral gain on demineralized dentin when using with hydroxyapatite nanoparticle.

\section{INTRODUCTION}

Tooth demineralization results from exposure to acids, from bacterial origin (caries) or dietary $\backslash$ gastric sources (dental erosion) which causes gradual but irreversible loss of tooth structure. The management of dental caries involves a range of different strategies, all of which have their inherent limitations. One of these strategies is the prevention by controlling dietary habits, oral hygiene, plaque control and the use of topical fluoride; dependent on total patient compliance ${ }^{(1-3)}$.

Different dentine remineralization strategies have been investigated, most of which focus on the use of bioactive glass ,fluoride -releasing materials, casein phosphopeptide - amorphous calcium phosphate complexes, artificial saliva solution, calcium hydroxide and Portland cement. An alternative strategy, that is becoming the focus of much research in this field, is the use of nanoparticles for the management of dental caries. Nano-sized calcium fluoride nanoparticulate hydroxyapatite, nano -sized carbonated apatite carbonate-hydroxy apatite nanocrystals and nano -particulate bioactive glass are among those nano-materials that seem to increase the mineral content of enamel and $\backslash$ or dentine ${ }^{(4)}$.

For the hydroxyl apatite crystal to perform more efficient remineralization, smaller particle size is achieved by using many solvents. One of these solvents is sodium hexa meta phosphate (SHMP). TEM examination revealed that infiltration of demineralized dentine with HA and SHMP was successful. The bulk dentine was found saturated with a cloud of less than $5 \mathrm{~nm}$ HA nanoparticles. The
SHMP crystals had distinct irregular shapes and were considerably larger than the HA particles with sizes in the micro-scale. However, the effect of the artificial saliva on the action of the remineralization of enamel and dentine is still to be educated and needs more effort in investigation ${ }^{(5-7)}$.

\section{MATERIALS AND METHODS}

\section{Selection of teeth}

Forty freshly extracted human premolars were used in this study. They should be free from caires, macroscopic cracks, abrasion or extensive staining, Each tooth was horizontally cut to separate the crown from the root.

\section{Classification of samples}

The teeth were assigned in 2 groups, where group one consist of (15) teeth (2 halves) not immersed in artificial saliva, while group two included the samples which immersed in artificial saliva, Each group was then subdivided into four subgroups of 5 samples each, according to the treatment solution used to the following:

Subgroup (A1): treated with hydroxyapatite nanoparticle

Subgroup (A2): treated with sodium hexametaphosphate,

Subgroup (A3): treated with combination of two materials $(\mathrm{HA}+\mathrm{SHMP})$.

\section{Preparation of materials}

\section{Preparation of hydroxyl apatite crystals solution (HAP):}

To prepare hydroxyl apatite solution with $0.03 \%$ concentration, $0.03 \%$ gram of hydroxyl apatite powder salt was weighted on balance sensor and dissolved by gentle shaking in prepration bottle, in $100 \mathrm{ml}$ distilled water.

The $\mathrm{pH}$ of the solution at $37^{\circ} \mathrm{C}$ was measured using $\mathrm{pH}$ meter ( $\mathrm{pH} 315 \mathrm{I} /$ SET, WTW Company, weilheim, Germany) that has $\mathrm{pH}$ and temperature sensor. 
The $\mathrm{pH}$ of the freshly prepared hydroxyl apatite solution was 2,3 at $37^{\circ} \mathrm{C}$, the solution was freshly prepared just prior to its use.

The preparation of hydroxyl apatite solution with 0.03 concentration, 0.03 gram of powder salt and dissolved in 50\% DW: $50 \%$ ethanol, ethanol is believed to disperse the HA agglomerates, this low concentration of solutions proved to be ideal as it enable distinct visualization of primary particles while avoiding clustering.

\section{Preparation of sodium hexametaphosphate solution (SHMP):}

To prepare sodium hexametaphosphate solution with concentration $0.03 \%, 0.03 \%$ gram of SHMP salt was weighted on balance sensor and dissolved by shaking of the preparation bottle, in $100 \mathrm{ml}$ distilled water.

\section{Preparation of combination materials solution:}

Prepare of 0.03 hydroxyapatite mixed with 0.01 sodium hexametaphosphate powder in a 50:50 distilled water DW to acetone solution.

\section{Scanning Electron Microscope:}

A representative sample from each group was chosen to examine the micro-morphological changes that occurred in enamel surface and in the dentin after etching and after the application of the remineralizing solutions. The specimens were glued, with the proximal surface facing upwards, on an scanning electron microscope (SEM) (JEOL JSM-5500 LV at 20KV) and examined at 4000x \&5000x \& $6000 x$. Then photo micrographed. The enamel surfaces of specimens were first sputter coated with a 3-nm- thick layer of gold (80\%) / palladium (20\%) prior to examinations.

\section{Energy dispersive X-ray spectrometric Analysis (EDXA):}

The sample of each subgroup were analyzed using Energy dispersive X-ray spectrometric Analysis (EDXA) to determine the calcium and phosphorous mineral content and the change in the levels of these elements percentage due to demineralization. As well, the fluoride content was deduced in respect to these subgroups treated with HA solutions. EDXA was performed using Joel model JSM 6510 LV installed on Scanning Electron Microscope (SEM), Thermo Electron System 300.

The sample was placed under vacuum and excited to a higher energy state with an electron beam. As the electrons of each element falls back down to its original energy state it emits X-ray energy at different specified wavelength. Each element identified by know wavelength on the $\mathrm{x}$-axis represented by a peak and the intensity of that peak on the Y-axis determine the amount of elements. EDXA is semiquantitative analysis with detection limits typically $1 \%$ or slightly higher so trace elements will not be detected.

\section{Statistical analysis}

Data were presented as mean and standard deviation values repeated measures analysis of variance (ANOVA) was used determine the effect of three different solution on tooth surface. Unpaired test was used to compare between mean value of enamel and dentin in all different group-AP value $<0.05$ was considered significant.

\section{RESULTS}

\section{Quantitative analysis}

\section{Comparison between all groups of Enamel and dentine:}

It was found that $\mathbf{H A}+\mathbf{S H M P}+\mathbf{A S})$ group in enamel recorded the highest total mean mineral contents $(10.946 \pm 5.21 \%)$, followed by (HA + AS) group in enamel $(10.89 \pm 6.1 \%)$, then group (HA+SHMP) in dentine $(10.6 \pm 5.9 \%)$, while the mean for HA in enamel group recorded the lowest total mean mineral contents $(9.542 \pm 5.9 \%)$. The difference between groups was statistically in significant ( $>0.05$ ) (Table 1),(Fig 1,2,3). 
Table (1) Mineral profile change results (\%) for experimental groups by using ANOVA test.

\begin{tabular}{|c|c|c|c|c|}
\hline & Variables & Mean $\pm S D$ & $P$ value (Enamel \& dentine) & $P$ value (all groups) \\
\hline \multirow{6}{*}{ Enamel } & $\boldsymbol{H A}$ & $9.542 \pm 5.9$ & \multirow{6}{*}{$0.8732^{\text {ns }}$} & \multirow{12}{*}{$0.8103^{\mathrm{ns}}$} \\
\hline & SHMP & $9.623 \pm 5.7$ & & \\
\hline & $H A+S H M P$ & $9.742 \pm 5.5$ & & \\
\hline & $H A+A S$ & $10.89 \pm 6.1$ & & \\
\hline & $S H M P+A S$ & $9.946 \pm 6.1$ & & \\
\hline & $H A+S H M P+A S$ & $10.946 \pm 5.21$ & & \\
\hline \multirow{6}{*}{ Dentine } & $\boldsymbol{H A}$ & $9.6 \pm 5.3$ & \multirow{6}{*}{$0.932^{\mathrm{ns}}$} & \\
\hline & SHMP & $10.2 \pm 6.2$ & & \\
\hline & $H A+S H M P$ & $10.6 \pm 5.9$ & & \\
\hline & $H A+A S$ & $9.9 \pm 6.1$ & & \\
\hline & $S H M P+A S$ & $10.2 \pm 6.1$ & & \\
\hline & $H A+S H M P+A S$ & $10.4 \pm 5.9$ & & \\
\hline
\end{tabular}

$n s ;$ in-significant $(p>0.05)$

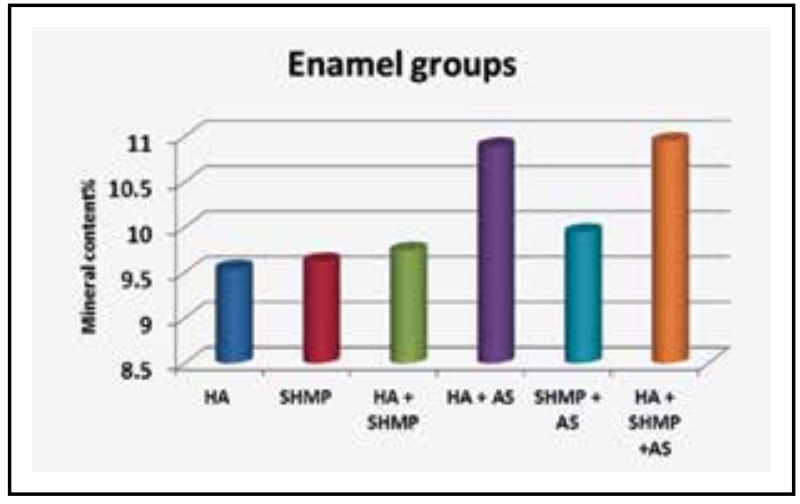

Fig. (1) The total mineral profile change (\%) for all experimental groups of enamel.

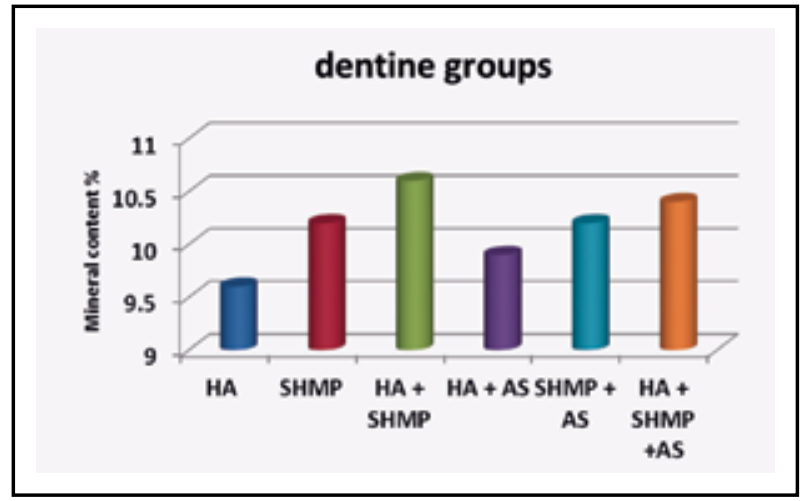

Fig. (2) The total mineral profile change (\%) for all experimental groups of dentin.

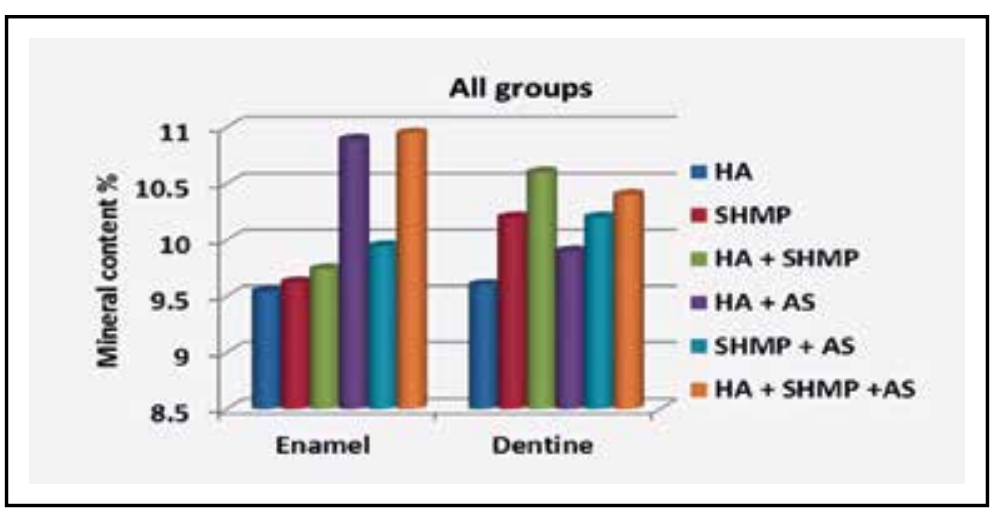

Fig. (3) The total mineral profile change (\%) for all experimental groups according to tooth structure. 


\section{Qualitative analysis}

\section{With Artificial Saliva}

On Enamel

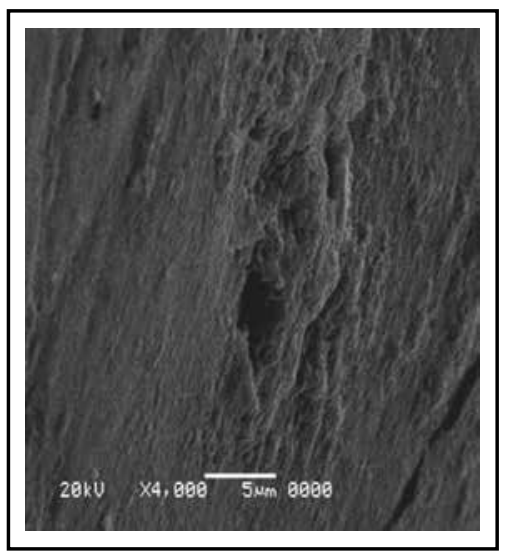

Fig. (4) SEM image for enamel after application of hydroxyapatite solution.

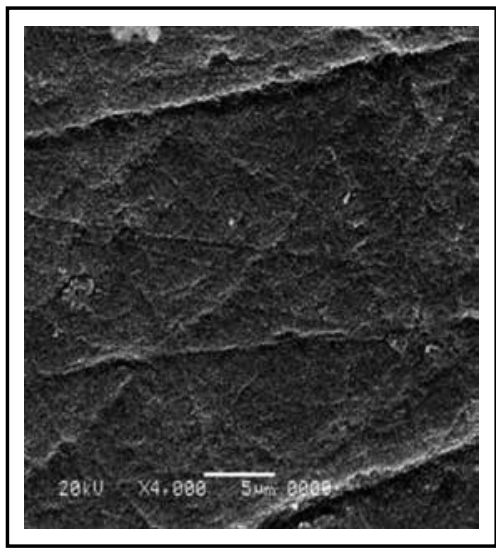

Fig. (6) SEM image for enamel after application of Sodium hexametaphosphate solution

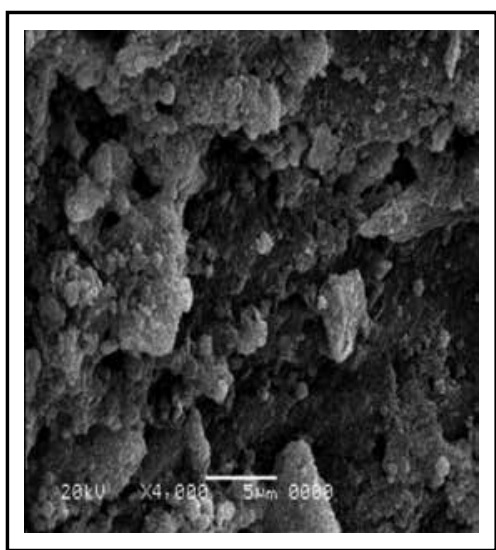

Fig(8) SEM image for Enamel surface after application of hydroxyapatite and Sodium hexametaphosphate solution

\section{Qualitative analysis \\ With Artificial Saliva}

\section{On Dentin}

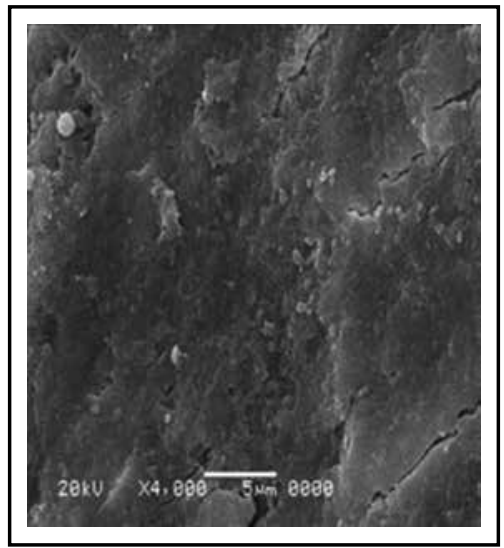

Fig. (5) SEM image for Dentin after application of hydroxyapatite solution.

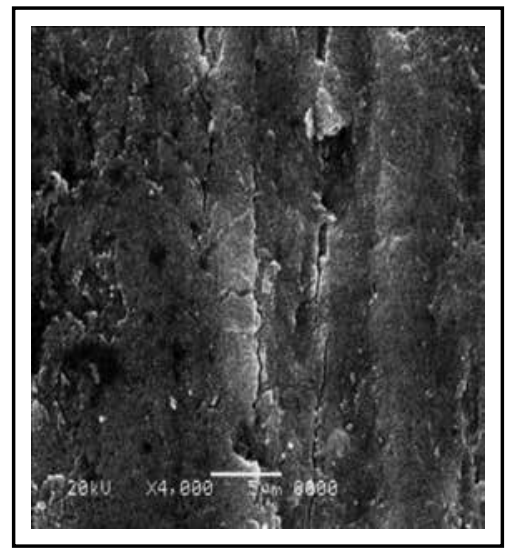

Fig. (7) SEM image for Dentin after application of Sodium hexametaphosphate solution

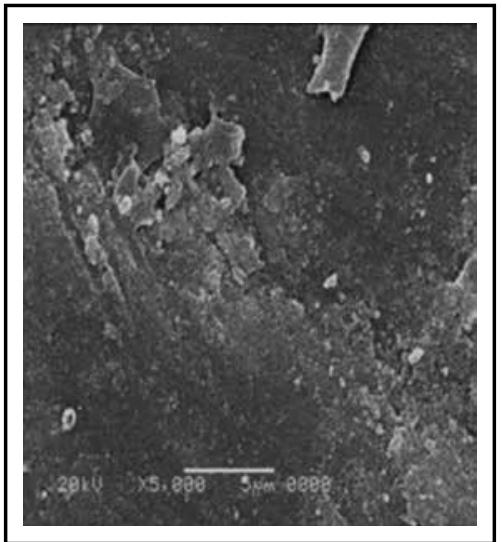

Fig.(9) SEM image for Dentin after application of hydroxyapatite and Sodium hexametaphosphate solution 


\section{With use of Artificial Saliva}

On Enamel

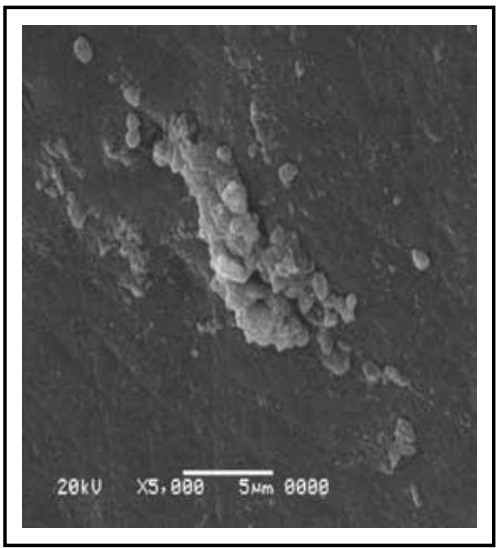

Fig. (10) SEM image showing Enamel surface after application of hydroxyapatite + artificial saliva solution

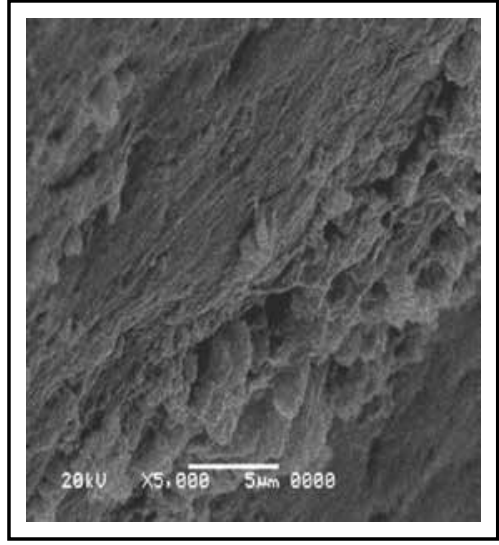

Fig. (12) SEM image for enamel surface after application of Sodium hexametaphosphateand artificial saliva solution

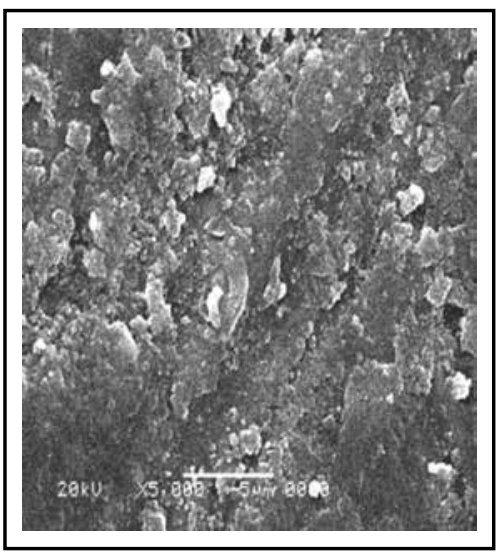

Fig. (14) SEM image for Enamel surface after application of hydroxyapatite and Sodium hexametaphosphate solution in present of artificial saliva

\section{With use of Artificial Saliva}

On Dentin

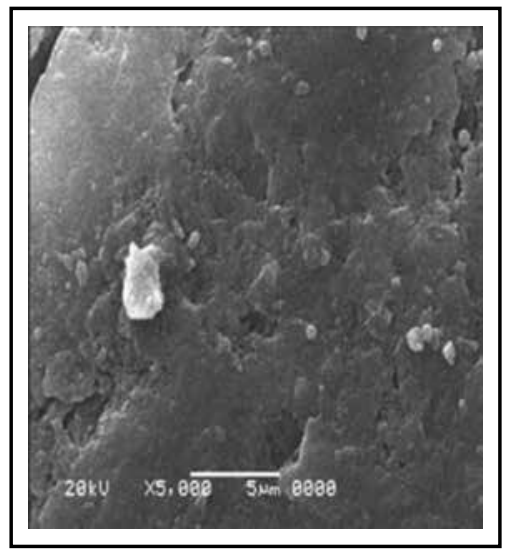

Fig. (11) SEM image showing Dentin surface after application of hydroxyapatite + artificial saliva solution

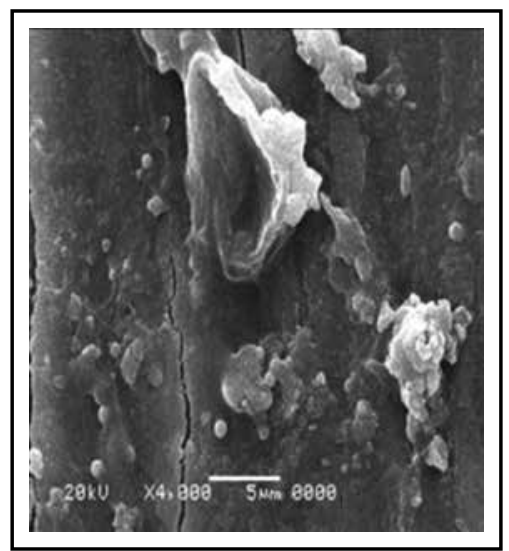

Fig. (13) SEM image for Dentin after application of Sodium hexametaphosphatewith artificial saliva solution

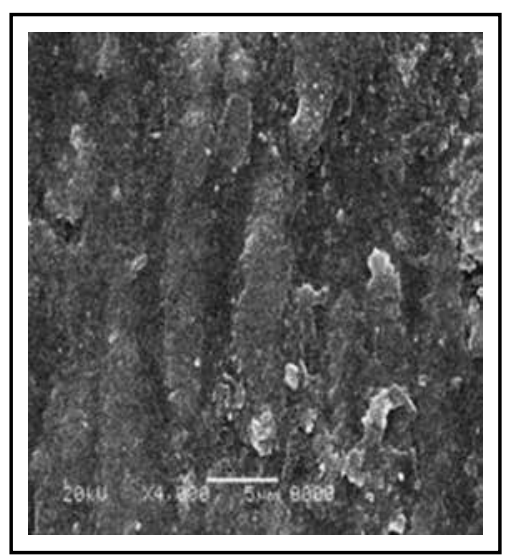

Fig. (15) SEM image for Dentin after application of hydroxyapatite and Sodium hexametaphosphate solution with artificial saliva 


\section{DISCUSSION}

Results revealed that that the infiltration of demineralized dentin with control nano-HA solutions the external surface of the dentine specimens were fully covered with a layer with nano-particles, but with poor infiltration deeply inside the collagen matrix, this is in agreement with, ${ }^{(I)}$ which said the large particle size, greater than the inter fibrillar spacings, of control nano - HA particles combined with there tendency to agglomerate are considered to be the cause of their reduced infiltration capability.

Results revealed that both enamel and dentin are affected by hydroxyapatite but on enamel it has recorded the lowest mineral gain mean value effect, this is in contradiction with ${ }^{(2)}$ that revealed that nano- hydroxyapatite could deposit strongly into the etched enamel surface under acidic solutions.

In agreement with previous study in which ${ }^{(3)}$ reported that the most of the materials used to treated dentin hypersensitivity usually aim to reduce stimuli conduction and apatite crystallization and possibly by remineralization. When hydroxyapatite is dissolved or abraded it cannot suddenly remineralize because enamel posses no cells.

Results revealed that control solutions of hydroxyapatite and sodium hexametaphosphate was the highest recover of the mineral loss, on dentin surface, this result was in contrast with ${ }^{(4)}$ which said there are two possible reasons why remineralization with biomimetic approach was incomplete within this surface region. The first is that water from the intrafibrillar compartment of the collagen fibrils was incompletely replaced by the newly formed apatite. and the other reason of incomplete remineralization of the surface region is the degradation of the collagen matrix.

Results revealed that sodium hexametaphosphate with artificial saline recored the low total mean mineral content on enamel and the highest total mean mineral content on dentine surface, the efficiency of particles to bind to the demineralized dentin matrix is considered to be highly dependent on biological factors such as the regulation exerted by non-collagenous protein. Notwithstanding, the nature of these interaction and bonds that may occur between them remains far from clear. Several of these proteins have been suggested to be associated with the regulation of mineral growth and deposition. Dentin phosphophoryn (DPP) is considered to be a regulator of the mineral phase nucleation within the dentin matrix ${ }^{(5 \& 6)}$ whereas dentin sialoprotein (DSP) is belived to regulate the initiation of dentin mineralization but not the maturation of mineralized dentin (7) It has been proposed that decorin and biglycan, two small leucine-rich proteoglycans, may also have a role in matrix-mediated formation of mineralized tissues including dentin. This is in disagreement with Adelisa et al ${ }^{(8)}$ who reported that the resin with SHMP and F presentsd the same ability of enamel remineralization when compared to fluoride resin without SHMP. And in agreement with. ${ }^{(9)}$ have shown significant reductions in hydroxyapatite crystal growth and mineralization of plaque in the presence of sodium hexameta phosphate. However this is in agreement with several studies ${ }^{(10-12)}$ who that revealed SHMP seems to act on the lesions area by facilitating the flux of calcium into the deeper enamel layers and reducing acid diffusion inside the enamel, once adsorbed on enamel, the SHMP probably will be retain charged ions of $\mathrm{CaF}^{+}$and $\mathrm{Ca}$ ${ }^{++}$by replacing $\mathrm{Na}^{+}$from cyclic structure. The interaction of $\mathrm{Ca}^{2+}$ with one or more SHMP leads the reticular formation on enamel also containing $\mathrm{CaF}^{+}$. The release of fluoride can increases calcium and phosphate in the biofilm and the presence of SHMP can retain such ions close to the enamel.

Results revealed a positive role of saliva to inhance remineralization in enamel and dentin, this is in agreement with Sensabaugh and Sagel ${ }^{(13)}$ who said fluoride, in various forms, is well recognized for its ability to faster remineralization of partialy demineralized tooth enamel using the calcium and 
phosphate present in saliva and anther study reported the 7- days pretreatment of enamel in a solution containing the same concentrations of calcium and phosphate present in the original whole saliva did not impart resistance against supsequent demineralization Featherstone et al ${ }^{(14)}$.

In contradication, ${ }^{(15)}$ reported that after free calcium and phosphate were removed from whole saliva by dialysis, the remaining organic molecules imparted the same resistance to demineralization as whole saliva.

On loading manner results revealed that sodium hexametaphosphate and hydroxyapatite was recover the mineral loss on dentin but statistically non - significantly ( $\mathrm{p}>0.05)$, this is in contradiction, ${ }^{(I)}$ reported that infiltration of demineralized dentine with this solutions was successful, the use of deflocculating agent SHMP improved the infiltration of hydroxyapatite with evidence of dentin saturation supported by the EDS analysis, addition of sodium hexametaphosphate was found earlier to cause complete de-agglomeration of hydroxyapatite particles reducing their primary particle size, and the use of acetone, as ahydrophobic diluted, has a significant effect on the infiltrative ability of hydroxyapatite in solution, where acetone is added to solution to enable deeper penetration of demineralized collagen matrix, by displacing the water molecules, when the HA was diluted in distilled water the infiltration ability was low.

At static load results revealed that the use of hydroxyapatite alone did not recover the mineral loss as will as the use of sodium hexametaphosphate alone, This is in agreement with ${ }^{(l)}$ who reported that the infiltration of demineralized dentine with control HA solution was poor infiltrated deep inside the collagen matrix, because the large particle size greater than the inter-fibrillar spacing, this is in combined with their tendency to agglomerate are considered to be the cause of their reducing infiltrative capability.

\section{CONCLUSIONS}

Hydroxyapatite nanoparticle has a better effect on demineralized enamel when used with artificial saliva and Sodium hexametaphosphate has a significant role in mineral gain on demineralized dentin when using with hydroxyapatite nanoparticle while the Control hydroxyapatite solution recorded the lowest mineral precipitation and remineralization formed on surface of demineralized enamel dentin substrate.Its suggested that particle size plays a major role in the degree of infiltration.

\section{REFERENCES}

1. Besinis $A$, Van Noort $\mathrm{R}$, and Martin $\mathrm{N}$ : Infiltration of demineralized dentine with silica and hydroxyapatite nanoparticles. Dent Mater. 2012 Sep; 28(9):1012-23.

2. Liu D, Yang Q Troczynski T and Tseng W. Structural evaluation of sol-gel - derived hydroxyapatite. Biomaterials 2002; 23(7):1679-87

3. Richard D, Trushkowsky and Anabella Oquendo. Treatment of Dentin Hypersensitivity. Dent Clin North Am. 2011 Jul; 55(3):599-608.

4. Yan Liu, Nan Li, Yipi Qi, Li-na Niu, Sally Elshafiy, Jing Mao, Lorenzo Breschi, David H, Pashley, Franklin R, Tay. The use of sodium trimetaphosphate as biomimetic analog of matrix phosphoproteins for remineralization of artificial caries-like dentin. Dent Mater. 2011;27(5):465-77

5. Lussi A, Crenshaw MA, Linde A. Induction and inhibition of hydroxyapatite formation by rat dentine phosphoprotein in vitro. Arch Oral Biol. 1988;33(9):685-91.

6. Traub W, Jodaikin A, Arad T, Veis A and Sabsay B.Dentin phosphophoryn binding to collagen fibrils. Matrix 1992;12(3):197-201.

7. Suzuki S, Sreenath T, Haruyama N, Honeycutt C, Terse $A$ and Cho A. Dentin sialoprotin and phosphoprotein have distinct roles in dentin mineralization. Matrix Biol. 2009;28(4):221-9.

8. Tiveron ARF, Delbem ACB, Gaban, Sassaki GK and Pedrini D. In vitro enamel remineralization capacity of composite resins containing sodium trimetaphosphate and fluoride. Clin Oral Investig. 2015 Jan 23

9. White DJ, Cox ER, Suszcynsky-Meister EM, Baig AA. In vitrostudies of the anticalculus efficacy of a sodium 
hexametaphosphate whitening dentifrice. J ClinDent. 2002; 13:33-37.

10. Takeshita EM, Exterkate RAM, Delbem ACB and ten Cate JM . Evaluation of different fluoride concentrations supplemented with trimetaphosphate on enamel De- and remineralization in vitro. Caries Res 2011;(45):494-497.

11. Ferreira L, Pedrini D, Okamoto AC m Jardim EG Jr, Henriques TA, Cannon M, Delbem ACB. Biochemical and microbiological characteristics of in situ biofilm formed on materials containing fluoride or amorphous calcium phosphate. Am J Dent. 2013;26(4):207-13.

12. Cochrane HJ,Saranathan S, Cai F, Cross KJ, Reynolds EC.
Enamel subsurface lesion remineralisation with casein phosphpeptide stabilized solutions of calcium $m$ phosphate and fluoride. Caries Res. 2008;42(2):88-97.

13. Sensabaugh $\mathrm{C}$ and Sagel ME. Stannous Fluoride Dentifrice with Sodium Hexametaphosphate. J Dent Hyg. 2009;83(2):70-8.

14. Ten Cate JM and Featherstone JDB. Mechanistic Aspects of the Interactions between Fluoride and Dental Enamel. Crit.Rev. Oral Biol.Med 1991; 2:283-296.

15. Featherstone JDB, Behrman JM and Bell JE. Effect of whole Saliva Components on Enamel Demineralization In Vitro. Crit Rev Oral Biol Med. 1993;4(3-4):357-62. 